\title{
Insertion of isocyanides into zirconium-alkyl bonds of di-ansa-zirconocene complexes. X-ray molecular structure of $\left.\left[\mathrm{Zr}^{\prime}\left(\mathrm{SiMe}_{2}\right)_{2}\left(\eta^{5}-\mathrm{C}_{5} \mathrm{H}_{3}\right){ }_{2}\right\} \mathrm{Cl}\left\langle\eta^{2}-\mathrm{C}(\mathrm{i}-\mathrm{Pr}) \mathrm{N}\left(2,6-\mathrm{Me}_{2} \mathrm{C}_{6} \mathrm{H}_{3}\right)\right\rangle\right]$
}

\author{
Antunio M. Barriola a , Ana M. Cano ${ }^{b}$, Tomás Cuenca ${ }^{b}$, Francisco J. Fernández ${ }^{b}$, \\ Pilar Gómez-Sal ${ }^{b .1}$, Antonio Manzanero' ${ }^{b}$, Pascual Royo ${ }^{\text {b. * }}$ \\ apartamento de Química. Universidad Simón Bolícar, Caracas, Venezuela \\ 'Departamento de Química Inorgánica. Unic ersidad de Alcalá. Campus Unicersitario, E-2887I Alcalá de Henares. Spain
}

Received 27 November 1996; revised 29 January 1997

\begin{abstract}
New dicyclopentadienyl iminoacyl zirconium complexes have been prepared and characterized by NMR spectroscopy. The reaction of $\left.\left[\mathrm{Zr}\left(\mathrm{SiMe}_{2}\right)_{2}\left(\eta^{3}-\mathrm{C}_{5} \mathrm{H}_{3}\right)_{2}\right] \mathrm{Me}_{2}\right]$ with CNR $\left(\mathrm{R}=2.6-\mathrm{Me}_{2} \mathrm{C}_{6} \mathrm{H}_{3}, \mathrm{t}-\mathrm{Bu}\right)$ yields $\left.\left.\left[\mathrm{Zr}(\mathrm{SiMe})_{2}\right)_{2}\left(\eta^{5}-\mathrm{C}_{5} \mathrm{H}_{3}\right)_{2}\right] \mathrm{Me}\left(\eta^{2}-\mathrm{CMeNR}\right)\right](\mathrm{R}=2.6-$ $\mathrm{Me}_{2} \mathrm{C}_{n} \mathrm{H}_{3}$, t-Bu), which reacts with a stoichiometric amount of water to give the $\mu-0 x \operatorname{dimers}^{2}\left[\mathrm{Zr}\left(\mathrm{SiMe}_{2}\right)_{3}\left(\eta^{5} \cdot \mathrm{C}_{5} \mathrm{H}_{3}\right)_{2}\right)\left(\eta^{2} \cdot\right.$ CMeNR) $]_{2}(\mu-O)\left(R=2.6-\mathrm{Me}_{2} \mathrm{C}_{6} \mathrm{H}_{3}\right.$, t-Bu). The chloro neophyl complex $\left.\left[\mathrm{Zr}\left(\mathrm{SiMe}_{2}\right)_{2}\left(\eta^{3}-\mathrm{C}_{5} \mathrm{H}_{3}\right)_{2}\right) \mathrm{Cl}\left(\mathrm{CH}_{2} \mathrm{CMe}{ }_{2} \mathrm{Ph}\right)\right]$ and other $\mathrm{B}-\mathrm{hy}$ drogen containing airconium chloro alkyls $\left.\left[\mathrm{Zr}\left(\mathrm{SiMe}_{2}\right)_{2}\left(\eta^{9} \mathrm{C}_{3} \mathrm{H}_{3}\right)_{2}\right) \mathrm{CIR}\right]\left(\mathrm{R}=\mathrm{Et}, \mathrm{n} \cdot \mathrm{Pr}\right.$, i-Pr) react with $\mathrm{CN}\left(2,6-\mathrm{Me} \mathrm{C}_{2} \mathrm{C}_{3}\right)$ to yield the relisted chloro iminoacyl complexes $\left[\mathrm{Zr}\left(\left(\mathrm{SiMe}_{2}\right)_{2}\left(\eta^{5}-\mathrm{C}_{5} \mathrm{H}_{3}\right)_{2}\right) \mathrm{Cl}\left(\eta^{2}-\mathrm{CRN}\left(2,6-\mathrm{Me}_{2} \mathrm{C}_{6} \mathrm{H}_{3}\right)\right\}\right](\mathrm{R}=\mathrm{Et}$, $\mathrm{n}-\mathrm{Pr}$, i-Pr). whereas no reaction was observed when $\mathrm{CN}(\mathrm{t}-\mathrm{Bu})$ was used. All the new iminoacyl complexes were characterized by ' $H$ and ${ }^{13} \mathrm{C}$ NMR spectroscopy and the

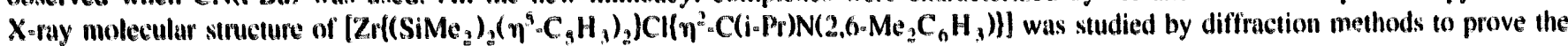
expected 'inside' coordination of the iminoacyl nitrogen atom. (C) $199 \%$ Elsevier Science $S . A$.
\end{abstract}

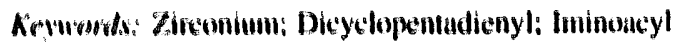

\section{Introduction}

Migratory insertion of carbon monoxide and the isoelectronic isocyanide ligands is one of the most simple reactions in organometallic chemistry, but very useful in many stoichiometric and catalytic processes [1]. Reaction of many Group 4 metal-alkyls with different alkyl and aryl isocyanides has enabled the isolation and characterization of a large number of $\eta^{2}$-iminoacyl complexes [2]. We have recently reported extensive studies on the isolation and reactivity of $\eta^{2}$-iminoacyl tantalum complexes [3]. Several studies related to the insertion of isocyanides into dicyclopentadienyl zirconium-alkyls have also been reported [4]. The 'inside' or 'outside' coordination of the oxygen atom in acyl ligands generated by the carbonylation of organozirconocenes has been extensively studied [5-7] and energy profiles for

\footnotetext{
Corresponding author.

$1 \mathrm{X}$-ray diffraction studies.
}

coordination of $\mathrm{CO}$ and further isomerization have been estimated [5]. Analogous studies have also been ex tended to the insertion of isocyanides and the inside or outside coordination of the nitrogen atom in $\eta^{2}$ aimin. oacyl compounds $[8-10]$. Here we report the insertion of CNR $\left(\mathrm{R}=\mathrm{t}-\mathrm{Bu}, 2,6 \cdot \mathrm{Me}_{2} \mathrm{C}_{6} \mathrm{H}_{3}\right)$ into the zirconiumalkyl bonds of $\left[\mathrm{Zr}\left(\left(\mathrm{SiMe}_{2}\right)_{2}\left(\eta^{5}-\mathrm{C}_{5} \mathrm{H}_{3}\right)_{2}\right) \mathrm{RX}\right](\mathrm{X} \equiv \mathrm{R}$ $\left.\mathrm{Me} ; \mathrm{X}=\mathrm{Cl}, \mathrm{R}=\mathrm{Me}, \mathrm{CH}_{2} \mathrm{CMe}_{2} \mathrm{Ph}, \mathrm{Et}, \mathrm{n}-\mathrm{Pr}, \mathrm{i}-\mathrm{Pr}\right)$ and the $X$-ray molecular structure of the i-propyl iminoacyl derivative.

\section{Results and discussion}

\subsection{Preparative results}

We have recently reported $[11,12]$ the alkylation of the dichloro zirconocene containing the doubly-bridged bis $\left(1,1^{\prime}, 2,2^{\prime}\right.$-dimethylsilanediyl) $-\eta^{5}$-dicyclopentadienyl ligand $\left[\mathrm{Zr}\left(\left(\mathrm{SiMe}_{2}\right)_{2}\left(\eta^{5}-\mathrm{C}_{5} \mathrm{H}_{3}\right)_{2}\right) \mathrm{Cl}_{2}\right]$ with lithium alkyls 
which leads to the $\beta$-hydrogen-free alkyl complexes $\left.\left[\mathrm{Zr}\left(\mathrm{SiMe}_{2}\right)_{2}\left(\eta^{\mathrm{s}}-\mathrm{C}_{5} \mathrm{H}_{3}\right)_{2}\right) \mathrm{RX}\right] \quad(\mathrm{X}=\mathrm{R}=\mathrm{Me} ; \mathrm{X}=\mathrm{Cl}$, $\mathrm{R}=\mathrm{CH}, \mathrm{CMe}, \mathrm{Ph})$. In similar reactions the much less stable $\beta$-hydrogen containing chloro-ethyl, $n$-propyl and i-propyl derivatives $\left[\mathrm{Zr}\left\{\left(\mathrm{SiMe}_{2}\right)_{2}\left(\eta^{5}-\mathrm{C}_{5} \mathrm{H}_{3}\right)_{2}\right\} \mathrm{ClR}\right](\mathrm{R}$ $=\mathrm{Et}$. $\mathrm{n} \cdot \mathrm{Pr}, \mathrm{i}-\mathrm{Pr})$ were also prepared [13].

All of these compounds reacted with isocyanides to give the corresponding iminoacyl complexes. Reaction of a toluene solution of the dimethyl zirconium compound $\left[\mathrm{Zr}\left(\left(\mathrm{SiMe}_{2}\right)_{2}\left(\eta^{5}-\mathrm{C}_{5} \mathrm{H}_{3}\right)_{2}\right\} \mathrm{Me}_{2}\right]$ with one equivalent of $\mathrm{CN}\left(2,6-\mathrm{Me}_{2} \mathrm{C}_{6} \mathrm{H}_{3}\right)$ at room temperature led to the methyl iminoacyl complex $\left[\mathrm{Zr}\left(\mathrm{SiMe}_{2}\right)_{2}\left(\eta^{5}\right.\right.$ $\left.\left.\left.\mathrm{C}_{5} \mathrm{H}_{3}\right)_{2}\right) \mathrm{Me}\left(\eta^{2}=\mathrm{CMeN}\left(2,6-\mathrm{Me}_{2} \mathrm{C}_{6} \mathrm{H}_{3}\right)\right\}\right]$ 1, which did not react when heated with further additions of the isocyanide, as expected for an 18 electron zirconium compound containing the $\eta^{2}$-coordinated iminoacyl group. Similar behaviour was observed when $\mathrm{CN}(\mathrm{t}-\mathrm{Bu})$ was used, leading to the iminoacyl complex $\left[\mathrm{Zr}\left(\left(\mathrm{SiMe}_{2}\right)_{2}\left(\eta^{5}-\mathrm{C}_{5} \mathrm{H}_{3}\right)_{2}\right) \mathrm{Me}\left(\eta^{2}-\mathrm{CMeN}(\mathrm{t}-\mathrm{Bu})\right)\right] 2$ (see Scheme 1). Both compounds 1 and 2 are extremely moisture sensitive and react with a stoichiometric amount of water to give the $\mu$-oxo dimers $\left.\left[\mathrm{Zr}\left(\mathrm{SiMe}_{2}\right)_{2}\left(\eta^{5}-\mathrm{C}_{3} \mathrm{H}_{3}\right)_{2}\right)\left(\eta^{2}-\mathrm{CMeNR}\right)\right]_{2}(\mu-\mathrm{O}) \quad(\mathrm{R}=$ 2.6-Me, $\mathrm{C}_{6} \mathrm{H}_{3}$ 3, $\mathrm{t}-\mathrm{Bu}$ 4). Likewise reaction of $\mathrm{CN}(\mathrm{t}-\mathrm{Bu})$ with the chloro neophyl complex under the same conditions led to the related chloro iminoacyl derivative $\left[\mathrm{Zr}\left(\mathrm{SiMe}_{3}\right)_{3}\left(\eta^{3}=\mathrm{C}_{3} \mathrm{H}_{3}\right)_{3}\right) \mathrm{Cl}\left(\eta^{3}=\mathrm{C}\left(\mathrm{CH}_{3} \mathrm{CMe}_{2} \mathrm{Ph}\right)[\mathrm{N}(2.6\right.$. $\left.\left.\left.\left.\mathrm{Me}_{2} \mathrm{C}_{6} \mathrm{H}_{3}\right)\right]\right\}\right]$.

Likewise addition of one equivalent of $\mathrm{CN}(2.6$.
$\mathrm{Me}, \mathrm{C}_{6} \mathrm{H}_{3}$ ) to toluene solutions of the $\beta$-hydrogen containing chloro-ethyl, n-propyl and i-propyl zirconium complexes at room temperature led to the related iminoacyl derivatives $\left[\mathrm{Zr}\left(\left(\mathrm{SiMe}_{2}\right)_{2}\left(\eta^{5}-\mathrm{C}_{5} \mathrm{H}_{3}\right)_{2}\right) \mathrm{Cl}\left(\eta^{2}\right.\right.$ $\left.\left.\mathrm{CR}\left(\mathrm{N}\left(2,6-\mathrm{Me}_{2} \mathrm{C}_{6} \mathrm{H}_{3}\right)\right\}\right)\right](\mathrm{R}=\mathrm{Et}$ 6, n-Pr 7, i-Pr 8), reactions being complete after stirring for $1 \mathrm{~h}$. The chloro-ipropyl complex was used in situ to avoid isomerization. All of the chloro iminoacyl compounds show a remarkable thermal stability and can be manipulated in air without transformation for short periods, as expected for rather inert 18 electron species.

\subsection{Structural characterization}

All of the new iminoacyl complexes 2-8 show the $\nu(\mathrm{CN})$ absorption band between 1575 and $1610 \mathrm{~cm}^{-1}$ in their IR spectra and the NMR behaviour expected for $C$. symmetrical molecules having equivalent cyclopentadienyl rings, but each showing non-equivalent ring protons and carbon atoms. Therefore their ' $H$ NMR spectra show four singlets between $\delta 0.39$ and $\delta 1.02$ for the four non-equivalent silicon bonded methyl groups located in the equatorial plane of symmetry along with the metal atom and the other three substituents, and three multiplets between $\delta 5.06$ and $\delta 6.90$ for the nonequivalent protons of the rings (see Section 3). The resonances due to the migrated alkyl group are shifted down-field with respect to the values observed for the starting metal bonded species. Similar behaviour is also
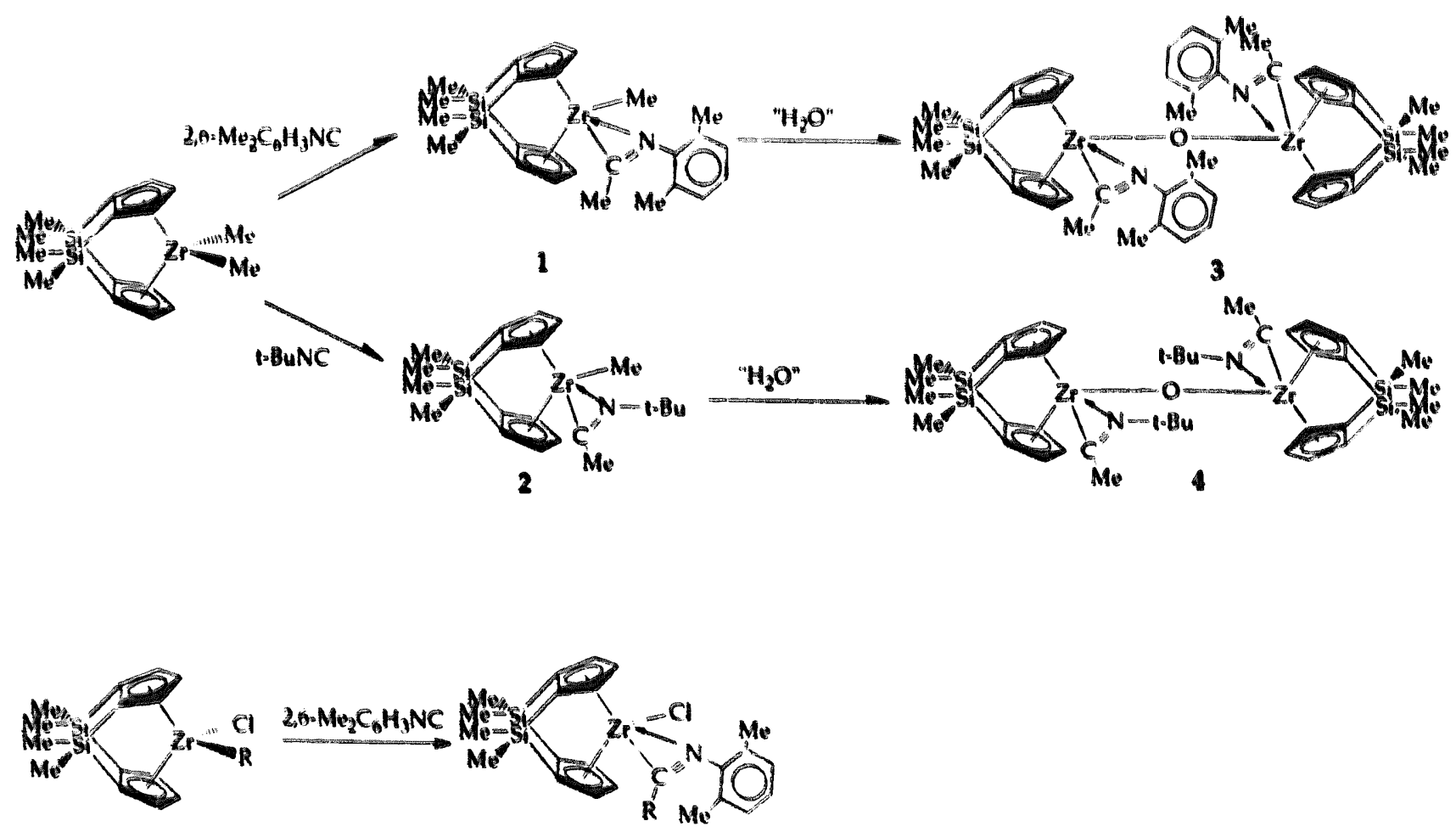

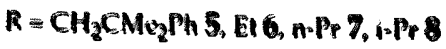




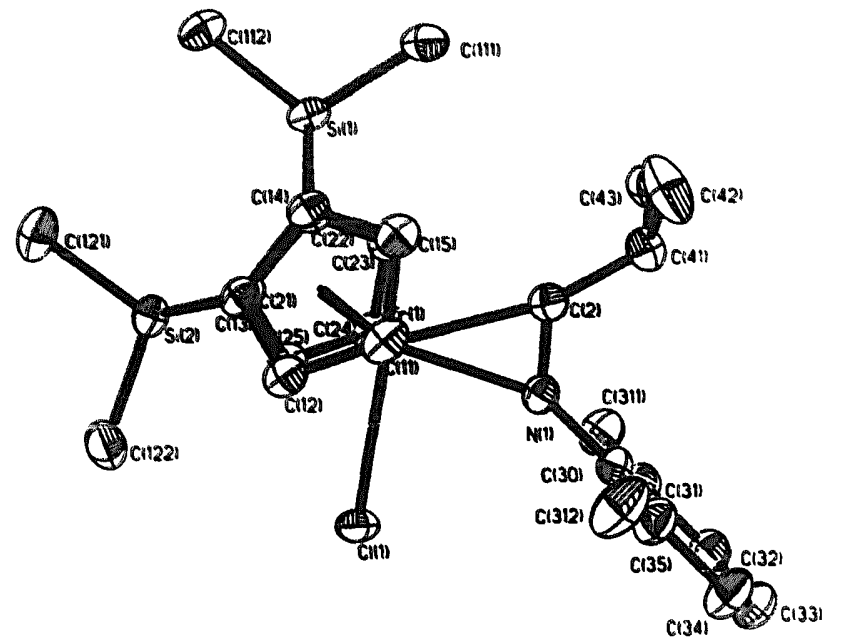

Fig. I. Molecular structure of 8.

observed in the ${ }^{13} \mathrm{C}$ NMR spectra, which show five signals for the ring carbon atoms and four resonances for the silicon carbon bonded atoms due to the chelating disposition of the dicyclopentadienyl ligand and the presence of three different substituents in the equatorial plane. Only one isomer is observed for all the new iminoacyl complexes. but from the spectral data it is not possible to distinguish the relative disposition of the two carbon and nitrogen coordinated atoms. For clarification we made a crystallographic study of the i-propyl compound $\left[\mathrm{Zr}\left((\mathrm{SiMe},)_{2}\left(\eta^{5}-\mathrm{C}_{5} \mathrm{H}_{3}\right)_{2}\right) \mathrm{Cl}\left(\eta^{2}-\mathrm{C}(\mathrm{i}-\mathrm{Pr}) \mathrm{N}(2.6-\right.\right.$ $\left.\left.\left.\mathrm{Me}_{2} \mathrm{C}_{6} \mathrm{H}_{3}\right)\right]\right] 8$.

Crystals of compound 8 suitable for X-riny diffraction studies were obtained from hexane. Fig. I shows the resulting molecular structure of 8 . Selected bond dis. tances and angles are presented in rable 1.

The molecular structure is a typicul metallocene system with the chlorine and the iminoacyl groups located aproximately in the plane bisecting the $\mathrm{Cp}-\mathrm{Zr}-\mathrm{Cp}$ unit. The coordination around the zirconium can be consid. ered tetrahedral if the cyclopentadienyl rings and the iminoacyl system are taken as occupying only one coordination site. The two $\mathrm{Cp}$ rings are bonded by two $\mathrm{SiMc}_{2}$ units leading to an eclipsed disposition. The central plane is defined by two silicon atoms from the doubly-bridged system and the metal atom. The $N(1)$ atom is located in this plane, but $\mathrm{Cl}(1)$ is displaced by $0.043 \AA$ and the $C(2)$ and $C(41)$ atoms are displaced by $0.062 \AA$ and $0.2 \AA$ respectively on the opposite side to the chorine. The i-propyl group is out of the plane.

The $\eta^{2}$-coordination of the iminoacyl group is sinuilar to that found in $\left[\mathrm{ZrCp}_{2}\left\{\eta^{2}-\mathrm{CMeN}(\mathrm{t}-\mathrm{Bu})\right\}\left(\mu-\mathrm{O}, \mathrm{O}^{\prime}\right.\right.$. $\left.\mathrm{O}_{2} \mathrm{CHCF}_{3}\right) \mathrm{ZrCp}_{2} \mathrm{Me}$ ] [7]. The $\mathrm{N}(1) \cdots \mathrm{C}(2)$ distance $1.268 \AA$ is consistent with a double bond and similar to the $1.255(5) \AA$ found in the above mentioned complex. in contrast to $\mathrm{N}-\mathrm{C}$ distances of $1.44 \AA$ corresponding to single bonds found for $\eta^{2}$-amino- and amido-alkyl complexes [14-16]. The $\mathrm{Zr}(1)-\mathrm{N}(1)$ distance 2.215(2) $\AA$ is quite similar to that found for the above mentioned dinuclear zirconium complex [2.216(4) $\AA$ ] and the $\mathrm{Zr}$ $\mathrm{C}(2)-\mathrm{N}(1)$ angle $71.3(2)^{\circ}$ is slightly smaller $\left(74.0(3)^{\circ}\right)$.

A more noticeable difference is observed in the $\mathrm{Zr}(1)-\mathrm{C}(2)$ distance $2.267(3) \AA$, clearly longer than in the iminoacyl compound $\left[\mathrm{ZrCp},\left\{\eta^{2}-\mathrm{CMeN}(\mathrm{t}-\mathrm{Bu})\right\}(\mu-\right.$ $\left.0, \mathrm{O}^{\prime}-\mathrm{O}_{2} \mathrm{CHCF}_{3}\right) \mathrm{ZrCp}_{2} \mathrm{Me}$ ] [7] 2.206(5) $\mathrm{\AA}$. This large distance in our case can probably be explained by the steric interaction of the methyl substituents of the i-propyl group with the $\mathrm{Cp}$ ring.

The nitrogen atom is located in an 'inside' position which is the more common for this sype of iminoacyl ligand. The $\mathrm{Zr}-\mathrm{Cl}$ distance is $2.532(1) \mathrm{A}$, longer than in the starting dichloro complex [11] where the distances are $2.427(1)$ and $2.428(1) \AA$. The two $C p$ rings are planar and the distances from $\mathrm{Zr}$ to the planes are 2.23.3(2) $\AA$ in both cases. The distances from the $\mathrm{Zr}$ to the $C$ atoms of the $C p$ rings range from $2.466(3) \AA$ to $2.611(3) \AA$, the distances to the carbon atoms bonded to silicon being shorter. This feature was also observed in

Tathe I

Selected bond lengths $(\AA)$ and bond angles $\left({ }^{\circ}\right)$ for compound 8

\begin{tabular}{|c|c|c|c|}
\hline $\operatorname{Zr}(1)-N(1)$ & $2.215(2)$ & $\mathrm{Zr}(1)=\mathrm{C}(2)$ & $2.267(3)$ \\
\hline $\mathrm{Zr}(1)-\mathrm{Cl}(1)$ & $2.5 .32(1)$ & $\mathrm{Zr}(1)-\mathrm{C}(11)$ & $2.611(3)$ \\
\hline$Z x(1)-C(12)$ & $2.560(.3)$ & $\mathrm{Zn}(1) \mathrm{C}(1,3)$ & $2.487(3)$ \\
\hline $\operatorname{Zr}(1)-C(14)$ & $2.487(.3)$ & $2+1)-C(15)$ & $2.563(3)$ \\
\hline$Z(1)-C(21)$ & $2.496(3)$ & $\operatorname{Zr}(1)-C(22)$ & $2.465(3)$ \\
\hline $2 r(1)-C(23)$ & $2.55+(3)$ & $21(1) C(2+)$ & $2023(3)$ \\
\hline $2 n(1)-C(25)$ & $2.5 \times 8(3)$ & $\operatorname{si}(1) C(111)$ & $1.856(4)$ \\
\hline $\mathrm{Si}(1)=\mathrm{C}(112)$ & $1.868(3)$ & $\mathrm{Sil}(1)-\mathrm{C}(22)$ & $1873(3)$ \\
\hline Sil 1$)-C(1+)$ & $1.887(3)$ & Sil 2$)(121)$ & $1.850(1)$ \\
\hline $\mathrm{Si}(2)=\mathrm{C}(122)$ & $1.854(4)$ & $\mathrm{Si}(2) \mathrm{C}(21)$ & $1.8 \times 2(3)$ \\
\hline $\operatorname{Sil}(2)-C(1,3)$ & $1.8 \times+(3)$ & $N(1) \quad(1)$ & $1.268(4)$ \\
\hline$N(1) \quad C(3(1))$ & 1 datkd) & $C(2) C(11)$ & $1.973(5)$ \\
\hline$C(+1)\left(+H_{2}\right)$ & $1.521(6)$ & $C(+1)-C(43)$ & $1.536(0)$ \\
\hline$C(31)-C(311)$ & $1.507(6)$ & $C(35)=C(312)$ & $1.912(6)$ \\
\hline $2 n(1)-C p 1$ & 2.230 & $\mathrm{ZN}(1) \mathrm{Cp}_{\infty}^{2}$ & 2389 \\
\hline $\mathrm{N}(1) \cdot \mathrm{ZN}(1) \mathrm{C}(\mathrm{I})$ & $32.8(1)$ & & \\
\hline$N(1)-\operatorname{Zr}(1)-C I(1)$ & $80.5 .3(7)$ & & \\
\hline$C(2)-Z+1(1)-C(1)$ & $11.3 .3 .3(8)$ & & \\
\hline$N(1)-C(2)-Z r(1)$ & $71.3(2)$ & & \\
\hline$N(1)-C(2)-C(+1)$ & $123.3(3)$ & & \\
\hline$C(+1)=C(2)-Z 1(1)$ & $16.5 .0(3)$ & & \\
\hline$C(3.5)-C(30)-N(1)$ & $119.6(.3)$ & & \\
\hline$C(.31)-C(.30)-N(1)$ & $119.1(3)$ & & \\
\hline$C(+2)-C(+1)=C(2)$ & $111.2(3)$ & & \\
\hline$C(42)=C(+1)=C(4,3)$ & $112.4(4)$ & & \\
\hline$C(2)=C(41)=C(4.3)$ & $110.7(3)$ & & \\
\hline$C(22)-S i(1)-C(14)$ & $91.4(1)$ & & \\
\hline$C(21)-\mathrm{Si}(2)-\mathrm{C}(1,3)$ & $92.4(1)$ & & \\
\hline$(p)-7 n(1)-C p^{2}$ & 118.0 & & \\
\hline$(1(1)-Z n(1)-C p 1$ & 105.7 & & \\
\hline $\mathrm{Cl}(1)-\mathrm{Zr}(1)-\mathrm{Cp})_{2}^{2}$ & 104.3 & & \\
\hline$C(2)-Z 1(1)=C p 1$ & 106.0 & & \\
\hline (2) $\mathrm{Zr}(1)-\mathrm{Cp}$ & $1(0), 1$ & & \\
\hline$N(1)-7,11)-C p 1$ & 119.1 & & \\
\hline$N(1)-7 x(1)-C p 2$ & 118.5 & & \\
\hline
\end{tabular}

Cpl is the centroid of C11. C12. C13. C14. C15.

Cp2 is the centroid of C21. C22. ( 233. C24. C25. 
other cases [11]. The angle between the two planes is $109.4(9)^{\circ}$ and the centroid- $\mathrm{Zr}$-centroid angle is $118.0^{\circ}$, slightly smaller than in the starting dichloro compound. The phenyl group is planar and is located almost perpendicular to the $\mathrm{Zr}-\mathrm{Si}(1)-\mathrm{Si}(2)$ plane (angle $\left.82.12(8)^{\circ}\right)$.

\section{Experimental}

All manipulations were performed under an inert atmosphere (argon) using Schlenk and high vacuum line techniques or a VAC Model HE 63P glovebox. Solvents were purified by distillation from an appropriate dry. ing/deoxygenating agent (sodium/benzophenone for diethyl ether. sodium for toluene. and sodium/potassium alloy for hexane).

The complexes $\left[\mathrm{Zr}\left(\mu-\left[\left(\mathrm{SiMe}_{2}\right)_{2}\left(\eta^{5}-\mathrm{C}_{5} \mathrm{H}_{3}\right)_{2}\right]\right\} \mathrm{Cl}_{2}\right]$ [11] and [Zrl $\left.\left(\mu-\left[\left(\mathrm{SiMe}_{2}\right)_{2}\left(\eta^{5}-\mathrm{C}_{5} \mathrm{H}_{3}\right)_{2}\right]\right) \mathrm{CIR}\right][11-13]$ were prepared according to literature procedures. $\mathrm{CN}(\mathrm{t}$ $\mathrm{Bu})$ and $\mathrm{CN}\left(2.6-\mathrm{Me}_{2} \mathrm{C}_{6} \mathrm{H}_{3}\right)$ (Aldrich) were obtained commercially.

NMR spectra were recorded on Varian Unity $\mathbf{3 0 0}$ and Varian Unity 500 Plus instruments $\left({ }^{1} \mathrm{H}\right.$ and ${ }^{13} \mathrm{C}$ chemical shifts were referenced to external $\mathrm{SiMe}_{4}, \delta$ 政 Oppm). IR spectra were performed in Nujol mulls on a Perkin-Elmer 883 spectrophotometer. Mass spectra were recorded on a Hewlett-Packard 5890 spectrometer. Elemental $\mathbf{C}$ and $\mathbf{H}$ analyses were carried out on a Perkin=Elmer $240 \mathrm{~B}$ microanalyser.

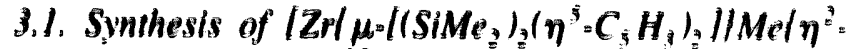 $\left.\mathrm{CMeN}\left(2,6 \mathrm{Me}_{2} \mathrm{C}_{\mathrm{A}} \mathrm{H}_{3}\right) /\right](1)$}

Toluene $(30 \mathrm{ml})$ was added to a mixture of $[\mathrm{Zr}(\mu=$ $\left.\left.\left[\left(\mathrm{SiMe}_{2}\right)_{2}\left(\eta^{3}-\mathrm{C}_{3} \mathrm{H}_{3}\right)_{3}\right]\right\} \mathrm{Me}_{2}\right](0.5 \mathrm{~g}, 1.37 \mathrm{mmol})$ and $\mathrm{CN}\left(2.6=\mathrm{Me}_{2} \mathrm{C}_{6} \mathrm{H}_{3}\right)(0.18 \mathrm{~g}, 1.37 \mathrm{mmol})$ at room temper ature and $i \mathrm{n}$ stirred for $1 \mathrm{~h}$. The solvent was com. pletely remov $d$ in vacuo to give a white-yellow solid. Recrystallization from hexane at $-20^{\circ} \mathrm{C}$ gave a white-yellow solid characterized as $1(0.64 \mathrm{~g}, 94 \%$ yield). Anal, Found: C, 60.41; H, 6.40; N, 3.01. $\mathrm{C}_{33} \mathrm{H}_{33} \mathrm{Si}_{2} \mathrm{ZrN}$ Calc.: C, 60.67; H, 6.72; $\mathrm{N}, 2.83 \%$. Mass spectrum $m / z ; M^{*}-(\mathbf{M e})(3 \%) ; M^{*}$ $-\left[\mathrm{CMe}=\mathrm{N}\left(2,6-\mathrm{Me}_{2} \mathrm{C}_{6} \mathrm{H}_{3}\right)\right](2 \%) ; \mathrm{M}^{+}-[\mathrm{CMe}=\mathrm{N}(2,6$ $\left.\left.\mathrm{Me}_{2} \mathrm{C}_{6} \mathrm{H}_{3}\right)\right]-(\mathrm{Me})(29 \%) ; M^{*}$ is not observed. IR $\nu_{1}(\mathrm{C}=\mathrm{N}): 1582 \mathrm{~cm}^{-1}$. 'H NMR $\left(\mathrm{C}_{6} \mathrm{D}_{6}\right): \delta 6.89(\mathrm{~m}, 3 \mathrm{H}$. $\left.\mathrm{C}_{6} H_{3}\right), \delta 6.81\left(\mathrm{~m}, 2 \mathrm{H}, \mathrm{C}_{5} H_{3}\right), \delta 6.32\left(\mathrm{~m}, 2 \mathrm{H}, \mathrm{C}_{5} H_{3}\right)$. $85.28\left(\mathrm{~m}, 2 \mathrm{H}, \mathrm{C}_{3} H_{3}\right), 1,95(\mathrm{~s}, 3 \mathrm{H}, M e-\mathrm{C} \equiv \mathrm{N}), \delta$ $1.70\left(\mathrm{~s}, 6 \mathrm{H}, 2.6 \mathrm{Me}_{2} \mathrm{C}_{8} \mathrm{H}_{3}\right), \delta 0.74(\mathrm{~s}, 3 \mathrm{H}, \mathrm{SiMe}), \delta$ $0.68\left(\mathrm{~s}, 3 \mathrm{H}, \mathrm{Si}_{\mathrm{M}} \mathrm{C}_{2}, \delta 0.63\left(\mathrm{~s}, 3 \mathrm{H}, \mathrm{Si} M \mathrm{C}_{2}\right), \delta 0.62(\mathrm{~s}\right.$. 3H, Si Me $)_{2}$, $0.12(\mathrm{~s}, 3 \mathrm{H}, \mathrm{ZrMe}),{ }^{13} \mathrm{C}$ NMR $\left(\mathrm{C}_{6} \mathrm{D}_{6}\right)$ : o $243.75(\mathrm{RN}=\mathrm{CMe}), \delta 144.81\left(C_{h} \mathrm{H}_{3}\right)$, \& 141.74 $\left(C_{5} \mathrm{H}_{3}\right), \delta 132.32\left(C_{3} \mathrm{H}_{3}\right), \delta 129.28\left(C_{6} \mathrm{H}_{3}\right), \delta 128.51$ $\left(C_{6} H_{3}\right), \delta 125.11\left(C_{6} H_{3}\right), \delta 122.24\left(C_{3} H_{3}\right), \delta 110.15$ $\left(C_{5} \mathrm{H}_{3}\right), \delta 107.50\left(C_{3} \mathrm{H}_{3}\right), \delta 24.84(\mathrm{RN}=\mathrm{CMe}), \delta$ $18.63\left(2,6-M_{2}-C_{5} H_{3}\right), \quad \delta \quad 7.41 \quad(\mathrm{ZrMe}), \quad \delta \quad 3.72$

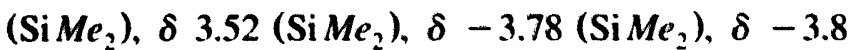
$\left(\mathrm{Si} \mathrm{Me}_{2}\right)$.

\subsection{Synthesis of $\left[\mathrm{Zr} / \mu-\left[\left(\mathrm{SiMe}_{2}\right)_{2}\left(\eta^{5}-\mathrm{C}_{5} \mathrm{H}_{3}\right)_{2}\right] / \mathrm{Me} / \eta^{2}-\right.$ CMeN(t-Bu) )] (2)}

Toluene $(30 \mathrm{ml})$ was added to a mixture of $[\mathrm{Zr} / \mu$ $\left.\left.\left[\left(\mathrm{SiMe}_{2}\right)_{2}\left(\eta^{5}-\mathrm{C}_{5} \mathrm{H}_{3}\right)_{2}\right]\right\} \mathrm{Me}_{2}\right](0.5 \mathrm{~g}, 1.37 \mathrm{mmol})$ and $\mathrm{CN}(\mathrm{t}-\mathrm{Bu})(0.15 \mathrm{ml}, 1.37 \mathrm{mmol})$ at room temperature and then stirred for $1 \mathrm{~h}$. The solvent was completely removed in vacuo to give a white-yellow solid. Recrystallization from hexane at $-20^{\circ} \mathrm{C}$ gave a microcrystalline solid characterized as $2(0.57 \mathrm{~g}, 93 \%$ yield $)$. Anal. Found: C. 56.50; H, 7.07: N. 3.01. $\mathrm{C}_{21} \mathrm{H}_{33} \mathrm{Si}_{2} \mathrm{ZrN}$ Calc.: C. $56.44 ; H .7 .44 ; N, 3.13 \%$. Mass spectrum $m / z: M^{+}-(\mathrm{Me})(17 \%) ; \mathbf{M}^{+}-\mathrm{Me}(21 \%) ; \mathbf{M}^{+}$ $-[\mathrm{CMe}=\mathrm{N}(\mathrm{t}-\mathrm{Bu})](12 \%) ; \mathrm{M}^{+}$is not observed. IR $\nu_{s}(C=N): 1624 \mathrm{~cm}^{-1}$. 'H NMR $\left(C_{6} D_{6}\right): \delta 6.81(\mathrm{~m}, 2 \mathrm{H}$, $\left.\mathrm{C}_{5} \mathrm{H}_{3}\right), \delta 6.17\left(\mathrm{~m}, 2 \mathrm{H}, \mathrm{C}_{5} \mathrm{H}_{3}\right), \delta 5.06\left(\mathrm{~m}, 2 \mathrm{H}, \mathrm{C}_{5} \mathrm{H}_{3}\right)$. $\delta 2.28(\mathrm{~s}, 3 \mathrm{H}, M e-\mathrm{C}=\mathrm{N}-\mathrm{R}), \delta 1.09\left(\mathrm{~s}, 9 \mathrm{H}, M e_{3} \mathrm{C}\right), \delta$ 0.75 (s, 3H, Si Me, ), $\delta 0.69$ (s, 3H, Si Me $), \delta 0.68$ (s, 3H. Si Me, ), $\delta 0.57$ (s, 3H. Si Me, ), $\delta 0.23$ (s, 3H, $\mathrm{ZrMe}) .{ }^{13} \mathrm{C}$ NMR $\left(\mathrm{C}_{6} \mathrm{D}_{6}\right): \delta 233.30(\mathrm{RN}=C \mathrm{CMe})$. $\delta$ $142.54\left(C_{5} \mathrm{H}_{3}\right), \delta 133.47\left(C_{5} \mathrm{H}_{3}\right), \delta 122.06\left(C_{5} \mathrm{H}_{3}\right), \delta$ $105.74\left(C_{5} \mathrm{H}_{3}\right), \delta 105.24\left(C_{5} \mathrm{H}_{3}\right), \delta 62.02\left(\mathrm{Me}_{3} C\right) . \delta$ $30.11\left(\mathrm{Me}_{3} \mathrm{C}\right), \delta 24.50(\mathrm{RN}=\mathrm{CMe}), \delta 7.24(\mathrm{ZrMe}), \delta$ $4.19(\mathrm{Si} \mathrm{Me}), \delta 4.11\left(\mathrm{Si} \mathrm{Me}_{2}\right), \delta-3.37\left(\mathrm{Si} \mathrm{Me_{2 }}\right), \delta$ $=3.58\left(\mathrm{Si} \mathrm{M} c_{2}\right)$.

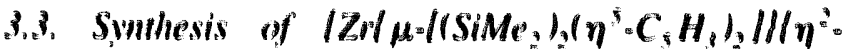
$C M e N\left(2,6=M_{0}, C_{n} H_{3}\right) \eta_{2}(\mu=O)(3)$

A solution of $\left[\mathrm{Zr}\left(\mu_{2}\left[\left(\mathrm{SiMc}_{3}\right)_{3}\left(\eta^{\overline{3}}-\mathrm{C}_{5} \mathrm{H}_{3}\right)_{2}\right] / \mathrm{Mc}\left(\eta^{\frac{3}{2}}=\right.\right.\right.$ $\left.\left.\operatorname{CMeN}\left(2,6-\mathrm{Me}_{2} \mathrm{C}_{6} \mathrm{H}_{3}\right)\right)\right]$ (1) $(0.35 \mathrm{~g}, 0.71 \mathrm{mmol})$ in toluene $(20 \mathrm{ml})$ at room temperature was stirred for $48 \mathrm{~h}$ with a stoichiometric amount of water $(0.007 \mathrm{ml}$. $0.35 \mathrm{mmol}$ ). The solvent was completely removed in vacuo to give a white-yellow solid characterized as 3 $(0.22 \mathrm{~g}, 64 \%$ yield). Anal. Found: C. 58.55: H. 5.98: N, 3.28. $\mathrm{C}_{48} \mathrm{H}_{601} \mathrm{Si}_{4} \mathrm{Zr}_{2} \mathrm{~N}_{2}$ Calc.: C. 59.08: H. 6.2; N. 2.87\%. Mass spectrum $m / \approx: \mathrm{M}^{+}-(\mathrm{Me})(3 \%): \mathrm{M}^{*}$ $-\left[\mathrm{CMe}=\mathrm{N}\left(2,6-\mathrm{Me}_{2} \mathrm{C}_{6} \mathrm{H}_{3}\right)\right](2 \%): \mathrm{M}^{+}-[\mathrm{CMe}=\mathrm{N}(2.6$. $\left.\mathrm{Me}_{2} \mathrm{C}_{6} \mathrm{H}_{3}\right)$ ]-(Me) (29\%): $\mathrm{M}^{+}$is not observed. IR $\nu_{1}\left(\mathrm{C}^{2}=\mathrm{N}\right): 1608 \mathrm{~cm}^{-1}$. 'H NMR $\left(\mathrm{C}_{6} \mathrm{D}_{6}\right): \delta 7.22(\mathrm{~m} .4 \mathrm{H}$, $\left.\mathrm{C}_{5} H_{3}\right) . \delta 6.90\left(\mathrm{~m}, 6 \mathrm{H}, \mathrm{C}_{\mathrm{m}} H_{3}\right), \delta 6.39\left(\mathrm{~m}, 4 \mathrm{H}, \mathrm{C}_{3} H_{3}\right)$. $\delta 5.48\left(\mathrm{~m}, 4 \mathrm{H}, \mathrm{C}_{3} H_{3}\right), \delta 1.91(\mathrm{~s}, 6 \mathrm{H}, \mathrm{Me}-\mathrm{C}=\mathrm{N}), \delta$ $1.84\left(\mathrm{~s}, 12 \mathrm{H}, 2,6-M e_{2} \mathrm{C}_{6} \mathrm{H}_{3}\right), \delta 0.97$ (s, 6H. Si Me $), \delta$ 0.69 (s, 6H, Si Me $), \delta 0.67$ (s, 6H, Si Me $), \delta 0.39$ (s, $\left.\left.6 \mathrm{H}_{1} \mathrm{SiMe}\right)_{2}\right){ }^{13} \mathrm{C} \cdot \mathrm{RMN}\left(\mathrm{C}_{6} \mathrm{D}_{6}\right): \delta 298.80(\mathrm{RN}=C \mathrm{CMe})$. $\delta 139.21\left(C_{5} \mathrm{H}_{3}\right), \delta 137.92\left(C_{5} \mathrm{H}_{3}\right) . \delta 130.54\left(C_{5} \mathrm{H}_{3}\right)$. $\delta 128.53\left(C_{5} H_{3}\right), \delta 124.91\left(C_{5} H_{3}\right), \delta 138.07\left(C_{6} H_{3}\right)$. $\delta 136.44\left(C_{6} \mathrm{H}_{3}\right), \quad \delta \quad 117.43\left(C_{6} \mathrm{H}_{3}\right), \quad \delta \quad 20.68$ $(\mathrm{RN}=\mathrm{CMe}), \quad \delta \quad 16.85 \quad\left(2,6-\mathrm{Me}_{2}-\mathrm{C}_{5} \mathrm{H}_{3}\right), \quad \delta \quad 0.36$ $\left(\mathrm{Si} \mathrm{Me}_{2}\right), \delta 0.19\left(\mathrm{Si} \mathrm{Me}_{2}\right), \delta-3.56(\mathrm{Si} \mathrm{Me}), \delta-7.57$ (Si $\left.\mathrm{Me}_{2}\right)$. 
3.4. Sinthesis of $\operatorname{Zr} / \mu-\left(\left(\operatorname{SiMe}_{2}\right)_{2}\left(\eta^{5}-C_{5} H_{3}\right)_{2}\right) / / \eta^{2}$ CMeN(t-Bu) $]_{2}(\mu-O)(4)$

A solution of $\left[\mathrm{Zr}\left(\mu-\left[\left(\mathrm{SiMe}_{2}\right)_{2}\left(\eta^{5}-\mathrm{C}_{5} \mathrm{H}_{3}\right)_{2}\right]\right\} \mathrm{Me}\left(\eta^{2}-\right.\right.$ $\mathrm{CMeN}(\mathrm{t}-\mathrm{Bu})\}]$ (2) $(0.35 \mathrm{~g}, 0.78 \mathrm{mmol})$ in toluene $(20 \mathrm{ml})$ at room temperature was stirred for $48 \mathrm{~h}$ with a stoichiometric amount of water $(0.007 \mathrm{ml}, 0.39 \mathrm{mmol})$. The solvent was completely removed in vacuo to give a white-yellow solid characterized as $4(0.22 \mathrm{~g}, 64 \%$ yield). Anal. Found: C, 54.39; H, 6.61; N, 3.39. $\mathrm{C}_{40} \mathrm{H}_{60} \mathrm{Si}_{4} \mathrm{Zr}_{2} \mathrm{~N}_{2}$ Calc.: C, 54.66; $\mathrm{H}, 6.82 ; \mathrm{N}, 3.19 \%$. Mass spectrum $m / z: \mathrm{M}^{+}$is not observed. IR $\nu_{\mathrm{s}}(\mathrm{C}=\mathrm{N})$ : $1600 \mathrm{~cm}^{-1}$. 'H NMR $\left(\mathrm{C}_{6} \mathrm{D}_{6}\right): \delta 7.20\left(\mathrm{~m}, 4 \mathrm{H}, \mathrm{C}_{5} \mathrm{H}_{3}\right), \delta$ $6.22\left(\mathrm{~m}, 4 \mathrm{H}, \mathrm{C}_{5} \mathrm{H}_{3}\right), \delta 5.28\left(\mathrm{~m}, 4 \mathrm{H}, \mathrm{C}_{5} \mathrm{H}_{3}\right), \delta 2.19(\mathrm{~s}$, $6 \mathrm{H}, M e-\mathrm{C}=\mathrm{N}-\mathrm{R}), \delta 1.12\left(\mathrm{~s}, 18 \mathrm{H}, M_{3} \mathrm{C}\right), \delta 1.02(\mathrm{~s}$, 6H. Si Me $e_{2}$, $\delta 1.00\left(\mathrm{~s}, 6 \mathrm{H}, \mathrm{Si} M e_{2}\right), \delta 0.69(\mathrm{~s}, 6 \mathrm{H}$,

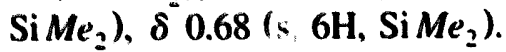

3.5. Symthesis of $\left.\mathrm{IZrCl} \mu-I\left(\mathrm{SiMe}_{2}\right)_{2}\left(\eta^{5}-C_{5} \mathrm{H}_{3}\right)_{2}\right] / / \eta^{2}-$ $\left.\left.\mathrm{ClCH}_{2} \mathrm{CMe}_{2}\left(\mathrm{C}_{6} \mathrm{H}_{5}\right)\right) \mathrm{N}\left(2,6-\mathrm{Me}_{2} \mathrm{C}_{6} \mathrm{H}_{3}\right)\right)(5)$

Toluene $(30 \mathrm{ml})$ was added to a mixture of $[\mathrm{Zr}(\mu$ $\left.\left.\left[\left(\mathrm{SiMe}_{2}\right)_{2}\left(\eta^{5} \cdot \mathrm{C}_{5} \mathrm{H}_{3}\right)_{2}\right]\right\} \mathrm{Cl}\left(\mathrm{CH}_{2} \mathrm{CMe}_{2} \mathrm{Ph}\right)\right] \quad(0.5 \mathrm{~g}$. $0.99 \mathrm{mmol})$ and $\mathrm{CN}\left(2.6-\mathrm{Me}_{2} \mathrm{C}_{6} \mathrm{H}_{3}\right)(0.13 \mathrm{~g}, 0.99 \mathrm{mmol})$ at room temperature and then stirred for $1 \mathrm{~h}$. The solvent was completely removed in vacuo to give a white-yellow solid. Recrystallization from hexane at $-20^{\circ} \mathrm{C}$ gave a white-yellow solid characterized as 5 $(0.59 \mathrm{~g}, 94 \%$ yield). Anal. Found: C, 62.56; H, 6.06; N, 2.83. $\mathrm{C}_{13} \mathrm{H}_{41} \mathrm{Si}, \mathrm{ZrNCl} \mathrm{Calc.:}$ C. 62.58; H. 6.32; N, 2.21\%. Mass spectrum $m / 2: M^{+}-[C M e=N(2,6-$ $\left.\left.\mathrm{Me}_{2} \mathrm{C}_{\mathrm{n}} \mathrm{H}_{3}\right)\right](14 \%)_{i} \mathrm{M}^{+}$is not observed. IR $\nu_{1}(\mathrm{C}=\mathrm{N})$ : $1580 \mathrm{~cm}^{1}$. H NMR $\left(\mathrm{C}_{6} \mathrm{D}_{0}\right): \delta 7.17\left(\mathrm{~m}, 5 \mathrm{H}, \mathrm{CMC}_{2} P h\right)$. $\delta 7.12\left(\mathrm{~m}, 2 \mathrm{H}, \mathrm{C}_{5} H_{3}\right), \delta 6.89\left(\mathrm{~m}, 3 \mathrm{H}, \mathrm{C}_{\mathrm{n}} H_{3}\right), \delta 6.23$ (III. 2H, C, $H_{3}$ ), $\delta 5.44\left(\mathrm{~m}, 2 \mathrm{H}, \mathrm{C}_{5} H_{3}\right), \delta 3.15(\mathrm{~s}, 2 \mathrm{H}$. $\left.\mathrm{C}_{2}\right), \delta 1.99\left(\mathrm{~s}, 6 \mathrm{H}, 2.6=\mathrm{Me}_{2} \mathrm{C}_{6} \mathrm{H}_{3}\right), \delta 1.05(\mathrm{~s}, 12 \mathrm{H}$. $\mathrm{CMe}, \mathrm{Ph}), \delta 0.98$ (s, 3H, Si Me, $), \delta 0.69$ (s, 3H. $\left.\mathrm{Si}_{\mathrm{Me}}{ }_{2}\right), \delta 0.64$ (s. 3H, Si Me $e_{2}$, $\delta 0.36$ (s, 3H, Si Me $e_{2}$ ). ${ }^{13} \mathrm{C}$ NMR $\left(\mathrm{C}_{6} \mathrm{D}_{6}\right): \delta 244.69(\mathrm{RN}=C \mathrm{CMe}), \delta 148.86$ $\left(C_{6} \mathrm{H}_{3}\right), \delta 142.74\left(C_{5} \mathrm{H}_{3}\right), \delta 129.60\left(C_{6} \mathrm{H}_{3}\right), \delta 128.89$ $\left(C_{6} \mathrm{H}_{5}\right), \delta 128.68\left(C_{6} \mathrm{H}_{5}\right), \delta 128.55\left(C_{6} \mathrm{H}_{3}\right), \delta 126.66$ $\left(C_{6} \mathrm{H}_{3}\right), \delta 125.88\left(C_{6} \mathrm{H}_{5}\right), \delta 125.74\left(C_{6} \mathrm{H}_{5}\right), \delta 124.52$ $\left(C_{5} \mathrm{H}_{3}\right), \delta 110.94\left(C_{5} \mathrm{H}_{3}\right), \delta 110.41\left(C_{5} \mathrm{H}_{3}\right), \delta 107.52$ $\left(C_{5} \mathrm{H}_{3}\right), \delta 30.32\left(\mathrm{CMe}_{2} \mathrm{Ph}\right), \delta 29.45\left(\mathrm{RN}=\mathrm{CCH}_{2}-\right)$. $\delta 19.71\left(2,6-\mathrm{Me}_{2}-\mathrm{C}_{6} \mathrm{H}_{3}\right), \quad \delta \quad 3.43(\mathrm{Si} \mathrm{Me}), \quad \delta \quad 3.14$ $\left(\mathrm{Si} M e_{2}\right), \delta-3.38\left(\mathrm{Si} M e_{2}\right), \delta-3.53\left(\mathrm{Si} M e_{2}\right)$.

3.6. Synthesis of $\operatorname{IZCl} \mu-/\left(\operatorname{SiMe}_{2}\right)_{2}\left(\eta^{5} \cdot \mathrm{C}_{5} \mathrm{H}_{3}\right)_{2} / \|\left(\eta^{2}\right.$. $\left.\mathrm{C} \mathrm{CH}_{2}-\mathrm{CH}_{3}\right) \mathrm{N}\left(2,6-\mathrm{Me}_{2} \mathrm{C}_{6} \mathrm{H}_{3}\right) /($ (6)

Toluene $(30 \mathrm{ml})$ was added to a mixture of $[\mathrm{Zr}(\mu$ $\left.\left.\left[\left(\mathrm{SiMe}_{2}\right)_{2}\left(\eta^{5}-\mathrm{C}_{5} \mathrm{H}_{3}\right)_{2}\right]\right\} \mathrm{Cl}\left(\mathrm{CH}_{2}-\mathrm{CH}_{3}\right)\right] \quad(0.5 \mathrm{~g}$. $1.25 \mathrm{mmol})$ and $\mathrm{CN}\left(2,6-\mathrm{Me}_{2} \mathrm{C}_{6} \mathrm{H}_{3}\right) \quad(0.164 \mathrm{~g}$, $1.25 \mathrm{mmol}$ ) at room temperature and then stirred for $1 \mathrm{~h}$. The solvent was completely removed in vacuo to give a white-y/ellow solid. Recrystallization from hexane at $-20^{\circ} \mathrm{C}$ gave a white solid characterized as $6(0.63 \mathrm{~g}$, 95\% yield). Anal. Found: C, 56.74; H, 6.18; N, 2.74 . $\mathrm{C}_{25} \mathrm{H}_{32} \mathrm{Si}_{2} \mathrm{ZrN}$ Calc.: C, 56.74; $\mathrm{H}, 6.05 ; \mathrm{N}, 2.64 \%$. Mass spectrum $\mathrm{m} / z:\left[\mathrm{M}^{+}\right]-(\mathrm{Et})(0.6 \%) ;\left[\mathrm{M}^{+}\right]-(\mathrm{Et}-$ CNR) (3\%); [Et-CNR] ${ }^{+}(60.6 \%)$. IR $\nu_{\mathrm{s}}(\mathrm{C}=\mathrm{N})$ : $1585 \mathrm{~cm}^{-1}$. 'H NMR $\left(\mathrm{C}_{6} \mathrm{D}_{6}\right): \delta 7.21\left(\mathrm{~m}, 2 \mathrm{H}, \mathrm{C}_{5} \mathrm{H}_{3}\right), \delta$ $6.89\left(\mathrm{~m}, 3 \mathrm{H}, \mathrm{C}_{6} H_{3}\right), \delta 6.60\left(\mathrm{~m}, 2 \mathrm{H}, \mathrm{C}_{5} \mathrm{H}_{3}\right), \delta 5.51(\mathrm{~m}$, $\left.2 \mathrm{H}, \mathrm{C}_{5} \mathrm{H}_{3}\right), \delta 2.40\left(\mathrm{q}, J=7.69 \mathrm{~Hz}, 2 \mathrm{H}, \mathrm{CH}_{2} \mathrm{CH}_{3}\right), \delta$ $1.88\left(\mathrm{~s}, 6 \mathrm{H}, 2,6-\mathrm{Me}_{2} \mathrm{C}_{6} \mathrm{H}_{3}\right), \delta 0.98\left(\mathrm{~s}, 3 \mathrm{H}, \mathrm{Si} M e_{2}\right), \delta$ 0.71 (s, 3H, Si Me $), \delta 0.68$ (s, 3H, Si Me $), \delta 0.65$ (t, $\left.J=7.69 \mathrm{~Hz}, 2 \mathrm{H}, \mathrm{CH}_{2} \mathrm{CH}_{3}\right), \delta 0.41\left(\mathrm{~s}, 3 \mathrm{H}, \mathrm{Si} \mathrm{Me}_{2}\right) .{ }^{13} \mathrm{C}$ $\operatorname{NMR}\left(\mathrm{C}_{6} \mathrm{D}_{6}\right): \delta 243.61(\mathrm{RN}=C \mathrm{CMe}), \delta 143.7\left(C_{6} \mathrm{H}_{3}\right)$, $\delta 141.9\left(C_{5} \mathrm{H}_{3}\right), \delta 129.4\left(C_{6} \mathrm{H}_{3}\right), \delta 128.5\left(C_{6} \mathrm{H}_{3}\right), \delta$ $125.6\left(C_{6} \mathrm{H}_{3}\right), \delta 123.9\left(C_{5} \mathrm{H}_{3}\right), \delta 111.4\left(C_{5} \mathrm{H}_{3}\right), \delta$ $109.5\left(C_{5} \mathrm{H}_{3}\right), \delta 108\left(C_{5} \mathrm{H}_{3}\right), \delta 31.6\left(-\mathrm{CH}_{2} \mathrm{CH}_{3}\right), \delta$ $19.1\left(2,6-\mathrm{Me}_{2}-\mathrm{C}_{6} \mathrm{H}_{3}\right), \delta 9.91\left(\mathrm{CH}_{2} \mathrm{CH}_{3}\right), \delta 3.3$ $\left(\mathrm{Si}_{\mathrm{Me}}\right), \delta 3.2\left(\mathrm{Si} \mathrm{Me}_{2}\right), \delta-3.6\left(\mathrm{Si} \mathrm{Me}_{2}\right), \delta-4.5$ $\left(\mathrm{Si} \mathrm{Me}_{2}\right)$.

3.7. Sinthesis of $\left.\mathrm{IrCl}(\mu-\mathrm{ISiMe})_{2}\left(\eta^{5}-\mathrm{C}_{5} \mathrm{H}_{3}\right)_{2}\right] / / \eta^{2}$. $\left.\left.\mathrm{Cl}_{\mathrm{CH}}-\mathrm{CH}_{2}-\mathrm{CH}_{3}\right) \mathrm{N}\left(2,6-\mathrm{Me}_{2} \mathrm{C}_{6} \mathrm{H}_{3}\right)\right]$ (7)

Toluene $(50 \mathrm{ml})$ was added to a mixture of $[\mathrm{Zr}\{\mu$ $\left.\left.\left[\left(\mathrm{SiMe}_{2}\right)_{2}\left(\eta^{5}-\mathrm{C}_{5} \mathrm{H}_{3}\right)_{2}\right]\right\} \mathrm{Cl}\left(\mathrm{CH}_{2}-\mathrm{CH}_{2}-\mathrm{CH}_{3}\right)\right] \quad(0.5 \mathrm{~g}$, $1.21 \mathrm{mmal})$ and $\mathrm{CN}\left(2,6 \cdot \mathrm{Me}_{2} \mathrm{C}_{6} \mathrm{H}_{3}\right)(0.158 \mathrm{~g}$, $1.21 \mathrm{mmol}$ ) at room temperature and then stirred for $\mathrm{lh}$. The solvent was completely removed in vacuo to give a white-yellow solid. Recrystallization from hexane at $-20^{\circ} \mathrm{C}$ gave a white solid characterized as $7(0.625 \mathrm{~g}$. 95\% yield). Anal. Found: C. 57.40; H, 6.32: N, 2.49. $\mathrm{C}_{26} \mathrm{H}_{34} \mathrm{Si}, \mathrm{ZrN}$ Calc.: C. 57.46: H. 6.30: N, $2.57 \%$. Mass spectrum $m / s:\left[\mathrm{M}^{+}\right]=(\mathrm{Pr})(2 \%):\left[\mathrm{M}^{+}\right]-(\mathrm{Pr}-$ CNR) $(7.8 \%) ; \quad[\mathrm{Pr}-\mathrm{CNR}]^{+}(100 \%)$. IR $\nu_{9}(\mathrm{CN}):$ $1585 \mathrm{~cm}^{-1}$. $H \mathrm{NMR}\left(\mathrm{C}_{n} \mathrm{D}_{6}\right): \delta 7.22\left(\mathrm{~m}, 2 \mathrm{H}, \mathrm{C}_{5} H_{3}\right)$. $6.89\left(\mathrm{~m}, 3 \mathrm{H}, \mathrm{C}_{6} H_{3}\right), \delta 6.62\left(\mathrm{~m}, 2 \mathrm{H}, \mathrm{C}_{5} H_{3}\right), \delta 5.52(\mathrm{~m}$. $\left.2 \mathrm{H}_{1} \mathrm{C}_{9} \mathrm{H}_{3}\right), \delta 2.47\left(\mathrm{~m}, 2 \mathrm{H}, \mathrm{CH}_{2} \mathrm{CH}_{2} \mathrm{CH}_{3}\right), \delta 1.91(\mathrm{~s}$. 6H. 2.6. $\left.\mathrm{Me}_{2} \mathrm{C}_{6} \mathrm{H}_{3}\right), \delta 1.27\left(\mathrm{~m}, 2 \mathrm{H}_{4} \mathrm{CH}_{2} \mathrm{CH}_{2} \mathrm{CH}_{3}\right), \delta$ 0.99 (s, 3H, Si Me $\left.e_{2}\right), \delta 0.72\left(\mathrm{~s}, 3 \mathrm{H}, \mathrm{Si} M \mathrm{C}_{2}\right), \delta 0.69$ (s, $\left.3 \mathrm{H}, \mathrm{Si} \mathrm{Me}_{2}\right), \delta 0.58\left(\mathrm{t}, 3 \mathrm{H}, \mathrm{CH}_{2} \mathrm{CH}_{2} \mathrm{CH}_{3}\right), \delta 0.43(\mathrm{~s}$, $\left.3 \mathrm{H}, \mathrm{Si} \mathrm{Me}_{2}\right)$. ${ }^{13} \mathrm{C}$ NMR $\left(\mathrm{C}_{6} \mathrm{D}_{6}\right): \delta 242.8(\mathrm{RN}=\mathrm{CMe}), \delta$ $143.9\left(C_{6} \mathrm{H}_{3}\right), \delta 142\left(C_{6} \mathrm{H}_{3}\right), \delta 129.6\left(C_{6} \mathrm{H}_{3}\right), \delta 128.6$ $\left(C_{6} \mathrm{H}_{3}\right), \delta 125.6\left(C_{6} \mathrm{H}_{3}\right), \delta 124.1\left(C_{5} \mathrm{H}_{3}\right), \delta 111.4$ $\left(C_{5} \mathrm{H}_{3}\right), \delta 109.4\left(C_{5} \mathrm{H}_{3}\right), \delta 108\left(C_{5} \mathrm{H}_{3}\right), \delta 40.8(-$ $\left.\mathrm{CH}_{2} \mathrm{CH}_{2} \mathrm{CH}_{3}\right), \delta 19.3\left(-\mathrm{CH}_{2} \mathrm{CH}_{2} \mathrm{CH}_{3}\right), \delta 19.2(2,6-$ $\left.\mathrm{Me}_{2}-\mathrm{C}_{6} \mathrm{H}_{3}\right), \delta 14.7\left(-\mathrm{CH}_{2} \mathrm{CH}_{2} \mathrm{CH}_{3}\right), \delta 3.3\left(\mathrm{Si} \mathrm{Me}_{2}\right)$, $\delta 3.2\left(\mathrm{Si} \mathrm{Me_{2 }}\right), \delta-3.6\left(\mathrm{Si} \mathrm{Me}_{2}\right), \delta-4.5\left(\mathrm{Si} \mathrm{Me_{2 }}\right)$.

3.8. Synthesis of $\left.\mathrm{IZCCl} \mu-/\left(\mathrm{SiMe}_{2}\right)_{2}\left(\eta^{5} \cdot \mathrm{C}_{5} \mathrm{H}_{3}\right)_{2}\right) /\left(\eta^{2}\right.$ $\mathrm{C}\left(\mathrm{CH}-\left(\mathrm{CH}_{3}\right)_{2}\right) \mathrm{N}\left(2,6-\mathrm{Me}_{2} \mathrm{C}_{6} \mathrm{H}_{3}\right) / /(8)$

A $2 \mathrm{M}$ solution of $\mathrm{MgCl}(\mathrm{i}-\mathrm{Pr})$ in THF $(1 \mathrm{ml}$, $1.98 \mathrm{mmol})$ at $-78^{\circ} \mathrm{C}$ was added to a solution of $\left[\mathrm{ZrCl}_{2}\left(\mu-\left(\mathrm{SiMe}_{2}\right)_{2}\left(\eta^{5}-\mathrm{C}_{5} \mathrm{H}_{3}\right)_{2}\right\}\right] \quad(0.8 \mathrm{~g}, 1.98 \mathrm{mmol})$ in THF $(25 \mathrm{ml})$ at $\mathrm{Ca}$. $-78^{\circ} \mathrm{C}$ and the mixture was stirred for $2 \mathrm{~h}$ at $10^{\circ} \mathrm{C}$. The solvent was completely removed in vacuo. $A$ solution of $\mathrm{CN}\left(2,6-\mathrm{Me}_{2} \mathrm{C}_{6} \mathrm{H}_{3}\right) \quad(0.26 \mathrm{~g}$. 
$1.98 \mathrm{mmol})$ in toluene $(50 \mathrm{ml})$ at room temperature was added to the mixture and then stirred for $1.5 \mathrm{~h}$. The solvent was completely removed in vacuo to give a white-yellow solid. Recrystallization from hexane at $-20^{\circ} \mathrm{C}$ gave a white-yellow crystalline solid characterized as $8(1.0 \mathrm{~g}, 94 \%$ yield). Anal. Found: C, 57.34; $\mathrm{H}$, 6.297; N, 2.77. $\mathrm{C}_{26} \mathrm{H}_{34} \mathrm{Si}_{2} \mathrm{ZrN}$ Calc.: C, 57.47; H, 6.30; N. $2.58 \%$. Mass spectrum $m / z:\left[\mathrm{M}^{+}\right]-$(i-Pr) $(4.6 \%)$; $\left[\mathrm{M}^{+}\right]-(\mathrm{i}-\mathrm{Pr}-\mathrm{CNR})(10.4 \%)$; [i-Pr-CNR] ${ }^{+}(100 \%)$. IR $\nu_{6}(\mathrm{CN}): 1575 \mathrm{~cm}^{-1}$. 'H NMR $\left(\mathrm{C}_{6} \mathrm{D}_{6}\right): \delta 7.23(\mathrm{~m}, 2 \mathrm{H}$, $\left.\mathrm{C}_{5} \mathrm{H}_{3}\right), \delta 6.89\left(\mathrm{~m}, 3 \mathrm{H}, \mathrm{C}_{6} \mathrm{H}_{3}\right), \delta 6.84\left(\mathrm{~m}, 2 \mathrm{H}, \mathrm{C}_{5} \mathrm{H}_{3}\right)$, $\delta 5.54\left(\mathrm{~m}, 2 \mathrm{H}, \mathrm{C}_{5} H_{3}\right), \delta 2.69(\mathrm{spt}, J=6.96 \mathrm{~Hz}, 1 \mathrm{H}$, $\left.\mathrm{CH}\left(\mathrm{CH}_{3}\right)_{2}\right), \delta 1.87\left(\mathrm{~s}, 6 \mathrm{H}, 2,6-\mathrm{Me}_{2} \mathrm{C}_{6} \mathrm{H}_{3}\right), \delta 1.02(\mathrm{~s}$, $\left.3 \mathrm{H}, \mathrm{Si} \mathrm{Me}_{2}\right), \delta 0.9\left(\mathrm{~d}, J=6.96 \mathrm{~Hz}, 6 \mathrm{H}, \mathrm{CH}\left(\mathrm{CH}_{3}\right)_{2}\right), \delta$ 0.73 (s, 3H, Si Me ),$\delta 0.68$ (s, 3H, Si Me $\left.e_{2}\right), \delta 0.48$ (s, 3H. Si $\left.M e_{2}\right) .{ }^{13} \mathrm{C}$ NMR $\left(\mathrm{C}_{6} \mathrm{D}_{6}\right): \delta 34.2(\mathrm{RN}=C \mathrm{CMe}), \delta$ $143.7\left(C_{6} \mathrm{H}_{3}\right), \delta \quad 142.5\left(C_{5} \mathrm{H}_{3}\right), \delta 129.5\left(C_{6} \mathrm{H}_{3}\right), \delta$ $128.4\left(C_{6} \mathrm{H}_{3}\right), \delta 125.5\left(C_{6} \mathrm{H}_{3}\right), \delta 122.5\left(C_{5} \mathrm{H}_{3}\right), \delta$

Table 2

Crystal data and structure refinement for compound 8

\begin{tabular}{|c|c|}
\hline $\begin{array}{l}\text { Empirical formula } \\
\text { Crystal size }\left(\mathrm{mm}^{3}\right) \\
\text { Colour } \\
\text { Crystal habit } \\
\text { Formula weight } \\
\text { Temperarure (K) }\end{array}$ & $\begin{array}{l}\mathrm{ZrClSi}_{2} \mathrm{NC}_{26} \mathrm{H}_{34} \\
\text { O., } \times 0.3 \times 0.3 \\
\text { Yellow-pale } \\
\text { Prismatic } \\
5,3,4 \\
293(2)\end{array}$ \\
\hline $\begin{array}{l}\text { Wavelengh }(\AA ̊) \\
\text { Crystal system }\end{array}$ & $\begin{array}{l}0,71073 \\
\text { Triclinic } \\
p \overline{1}\end{array}$ \\
\hline $\begin{array}{l}\text { Space group } \\
\text { Unil eell dimensions }\end{array}$ & $\begin{array}{l}\beta \bar{l} \\
a=9,053(1) \AA \\
b=11,949(1) \AA \\
r=12.397(1) \AA \\
a=108.38(1)^{\circ} \\
\beta=92.08(1)^{\circ} \\
\gamma=91.49(1)^{\circ}\end{array}$ \\
\hline $\begin{array}{l}\text { Volume }\left(\vec{A}^{3}\right) \\
Z \\
\text { Density (cale.) }\left(\mathrm{gcm}^{-1}\right) \\
\text { Absorption coefficient }\left(\mathrm{cm}^{-1}\right) \\
f(000) \\
\text { Pange for data collection }\left(^{\circ}\right) \\
\text { Index ranges }\end{array}$ & $\begin{array}{l}1336.7(2) \\
2 \\
1,350 \\
6.15 \\
364 \\
2,081024.97 \\
=11<h<11=14<1<13 . \\
0<1<14\end{array}$ \\
\hline $\begin{array}{l}\text { Reflections collected } \\
\text { Independent reflections } \\
\text { Reflections observed } \\
\text { with } I>20(I)\end{array}$ & $\begin{array}{l}4942 \\
4699\left(R_{\mathrm{imi}} 0.0212\right) \\
4095\end{array}$ \\
\hline $\begin{array}{l}\text { Absorption correction } \\
\text { Refinement method } \\
\text { Data/ nestraints/parameless } \\
\text { Goodness-of fit on } F^{2} \\
\text { Final } R \text { indices }[/>2 \text { of }(I)] \\
R \text { indices (all data) } \\
\text { Largest difference peak }\end{array}$ & $\begin{array}{l}N / A \\
F u l l=\text { matrix leasiosquares on } F \text { : } \\
4699 / 0 / 280 \\
1.085 \\
R_{1} \equiv 0.0378, w R_{2}=0.1019 \\
R_{1}=0.0470, w R_{2}=0.1084 \\
1.172 \text { and }=0.738\end{array}$ \\
\hline Weighting scheme (calc.) & $\begin{array}{l}w-\left[/ p^{2}\left(F_{v}^{2}\right)+(0.0745 p)^{2}+\right. \\
0.4 \| 15 P] \\
p=\left(F_{v}^{2}+2 F_{c}^{2}\right) / 3\end{array}$ \\
\hline
\end{tabular}

$110.9\left(C_{5} \mathrm{H}_{3}\right), \delta 110.5\left(C_{5} \mathrm{H}_{3}\right), \delta 107.8\left(C_{5} \mathrm{H}_{3}\right), \delta 38$ $\left(\mathrm{CH}\left(\mathrm{CH}_{3}\right)_{2}\right), \delta 19.5\left(\mathrm{CH}\left(\mathrm{CH}_{3}\right)_{2}\right), \delta 19.3\left(2,6-\mathrm{Me}_{2}-\right.$ $\left.\mathrm{C}_{6} \mathrm{H}_{3}\right), \delta 3.51\left(\mathrm{Si}_{M} e_{2}\right), \delta 3.36\left(\mathrm{Si} M e_{2}\right), \delta-3.04$ $\left(\mathrm{Si}_{\mathrm{Me}}\right), \delta-3.5\left(\mathrm{Si} M \mathrm{e}_{2}\right)$.

\subsection{X-ray structural determination for compound 8}

Crystallographic and experimental details of the crystal structure determinations are given in Table 2. Suitable crystals of complex 8 were mounted on an EnrafNonius CAD4 automatic four-circle diffractometer with bisecting geometry, equipped with a graphite-oriented monochromator and Mo $K \alpha$ radiation $(\lambda=0.71073 \AA)$. Data were collected at room temperature. Intensities were corrected for Lorentz and polarization effects in the usual manner. No absorption or extinction corrections were made.

The structure was solved by direct methods (SHELXS 90) [17] and refined by full-matrix least-squares against $F^{2}$ (sHELXL 93) [18]. All non-hydrogen atoms were refined anisotropically. In the last cycle of refinement the hydrogen atoms were positioned geometrically and refined using a riding model with fixed thermal parameters $\left(U=0.08 \AA^{2}\right)$.

Calculations were carried out on an ALPHA AXP (Digital) workstation.

\section{Supplementary material avallable}

Tables of anisotropic thermal parameters for non-hy. drogen atoms (Table 1), hydrogen coordinates (Table 2), coordinates for non=hydrogen atoms (Table 3), complete lists of bond lengths and angles (Table 4, 2 pages) and observed and calculated structure factors (11 pages).

\section{Acknowledgements}

We acknowledge the Dirección General de Investigaciơn Cientifica y Técnica (Spain) for financial support of this research (Project 92-0178-C). A.M. and F.J.F. are grateful to CAM and DGICYT respectively for fellowships. A.B. acknowledges BID-CONICIT (Venezuela) for financial support.

\section{References}

[1] L.D. Durfee, I.P. Rothwell. Chem. Rev, 88 (1988) 1059.

[2] L.R. Chamberlain, L.D. Durfee, P.E. Fanwick, L. Kobriger. S.L. Latesky, A.K.M. Mullen, I.P. Rothwell. K. Folting, J.C. Huff. man, W.E. Streib, R. Wang. J. Am. Chem. Soc. 109 (1987) 390.

[3] M.V. Galakhov, M. Gómes, G. Jimkinez, P. Royo, M.A. Pellinghelli, A. Tìripicchio, Organometallics 13 (1994) 1564: 14 (1995) 1901, 2843. 
[4] B.K. Campion, J. Falk. T.D. Tilley, J. Am. Chem. Soc. 109 (1987) 2049; M.F. Lappert. N.T. Luong-Thi, C.R.C. Melne. J. Organomet. Chem. 174 (1979) C35.

[5] K. Tatsumi, A. Nakamura, P. Hofmann. P. Stauffen. R. Hoffmann. J. Am. Chem. Soc. 107 (1985) 4440.

[6] G. Erker, F. Rosenfeldt. J. Organomet. Chem. 188 (1980) CI.

[7] P.J. Fagan, J.M. Manrquez, S.H. Vollmer, C.S. Day, V.W. Day, T.J. Marks. J. Am. Chem. Soc. 103 (1981) 2206.

[8] G.S. Bristow, M.F. Lappert, J.L. Atwood, W.E. Hunter, unpublished work (cited in D.J. Cardin, M.F. Lappert, C.L. Raston, Chemistry of Organo-zirconium and -hafnium Compounds, Halsted, New York, 1986. p. 223).

[9] B.D. Martin, S.A. Matchett, J.R. Norton, O.P. Anderson, J. Am. Chem. Soc. 107 (1985) 7952.

[10] T.V. Lubben, K. Plössl, J.R. Norton. M.M. Miller, O.P. Anderson. Organometallics $1 /$ (1992) 122.
[11] A. Cano, T. Cuenca, P. Gómez-Sal, B. Royo. P. Royo. Organometallics 13 (1994) 1688.

[12] A. Cano, T. Cuenca, P. Gómez-Sal, A. Manzanero, P. Royo. J. Organomet. Chem. 526 (1996) 227.

[13] F.J. Fernaindez. M.P. Gómez Sal. A. Manzanero, P. Royo, H. Jacobsen. H. Berke, Organometallics, 16 (1997) 1553.

[14] D.A. Gately, J.R. Norton, P.A. Goodson, J. Am. Chem. Soc. 117 (1995) 986.

[15] S.L. Buchwald, B.T. Watson, M.W. Wannamaker, J.C. Dewan, J. Am. Chem. Soc. 111 (1989) 4486.

[16] W.E. Hunter, D.C. Hmcir, R.V. Bynum, R.E. Penttila, J.L. Atwood, Organometallics 2 (1983) 750.

[17] G.M. Sheldrick. Acta Crystallogr. A46 (1990) 467.

[18] G.M. Sheldrick, sHElXı 93, University of Göttingen, Germany, 1993. 